\title{
PHASE DIFFERENCES BETWEEN INTENSITY AND DOPPLER SHIFT \\ AND BETWEEN TWO EUV EMISSION LINES OF SIII FOR 300 SECOND AND 95 SECOND CHROMOSPHERIC OSCILLATIONS
}

O.R. White and R.G. Athay

High Altitude Observatory, Boulder, Colorado 80307

Phase differences between intensity and doppler shift in each of the two bright emission lines of Sill at $\lambda 1816.93, \lambda 1817.45$ are determined for chromospheric oscillations with periods near 300 seconds and 95 seconds. Phase differences between the oscillations in the two lines are determined also.

For the 300 second oscillation, maximum intensity most often leads maximum blue shift by about 40 seconds and the oscillation in the fainter line at $\lambda 1817.45$ most often leads the oscillation in the stronger line at $\lambda 1816.93$ by about 35 seconds. For the 95 second oscillation, maximum intensity most often lags maximum blue shift by about 20 seconds and the oscillations in the fainter line most often lags the oscillations in the stronger line by about 12 seconds. The phase lags between the two lines together with the estimated height $s$ of formation are consistent with upward propagation with a phase velocity near the sound velocity for the 300 second oscillation and dowmward propagation with a phase velocity three to four times the sound velocity for the 95 second oscillation. The signs of the phase differences between maximum intensity and maximum blue shift are consistent with the direction of propagation if the waves are sound waves.

For both oscillations, examples can be found that are consistent with the opposite senses of propagation, viz., downward propagation for the 300 second oscillation and upward propagation for the 95 second oscillation. Less than a quarter of the cases fall within this category, however. 